\title{
Derivation of an Averaged Model of Isothermal Acoustics in a Heterogeneous Medium in the Case of Two Different Poroelastic Domains
}

\author{
A. A. Gerus*, S. A. Gritsenko**, and A. M. Meirmanov**** \\ Belgorod State University, ul. Pobedy 85, Belgorod, 308015 Russia \\ Received July 3, 2015
}

\begin{abstract}
We consider some mathematical model of isothermal acoustics in a composite medium consisting of two different porous soils (poroelastic domains) separated by a common boundary. Each of the domains has its own characteristics of the solid skeleton; the liquid filling the pores is the same for both domains. The differential equations of the exact model contain some rapidly oscillating coefficients. The averaged equations (i.e., without rapidly oscillating coefficients) are derived.
\end{abstract}

DOI: $10.1134 / \mathrm{S} 1990478916020058$

Keywords: composite media, periodic structure, Stokes isothermal equations, acoustics equations, poroelasticity, averaging of periodic structure, two-scale convergence

\section{STATEMENT OF THE PROBLEM}

Let the domain $Q$ under consideration be the unit cube $Q=(0,1) \times(0,1) \times(0,1)$; let a poroelastic medium fill the domain $\Omega=(0,1) \times(0,1) \times(0, a), 0<a<1$, and let the domain $G$ (another poroelastic medium) be the open complement of $\Omega$ :

$$
Q=\Omega \cup G \cup S^{(0)}, \quad S^{(0)}=\partial \Omega \cap \partial G .
$$

The motion of the mixture in $\Omega$ for $t>0$ is described by the system of equations

$$
\begin{gathered}
\left(\frac{\chi^{\varepsilon}}{\bar{c}_{f}^{2}}+\frac{1-\chi^{\varepsilon}}{\bar{c}_{s}^{2}}\right) p+\nabla \cdot \mathbf{w}=0, \\
\left(\rho_{f} \chi^{\varepsilon}+\left(1-\chi^{\varepsilon}\right) \rho_{s}\right) \frac{\partial^{2} \mathbf{w}}{\partial t^{2}}=\nabla \cdot \mathbf{P}+\rho^{\varepsilon} \mathbf{F}, \\
\mathbf{P}=\chi^{\varepsilon} \bar{\alpha}_{\mu} \mathbf{D}\left(x, \frac{\partial \mathbf{w}}{\partial t}\right)+\left(1-\chi^{\varepsilon}\right) \bar{\alpha}_{\lambda} \mathbf{D}(x, \mathbf{w})-p \mathbf{I},
\end{gathered}
$$

where $\chi^{\varepsilon}(\mathbf{x})$ is the characteristic function of the porous space $\Omega_{f}^{\varepsilon}$ in $\Omega, \chi^{\varepsilon}(\mathbf{x})=\chi(\mathbf{x} / \varepsilon) ; \bar{c}_{s}$ and $\bar{c}_{f}$ are the sound velocities in the solid and liquid parts, respectively; $\rho_{f}$ is the density of the liquid; $\rho_{s}$ is the density of the solid part; $\mathbf{F}$ is a given vector of the distributed mass forces; $l$ is the average size of pores; $L$ is the characteristic size of the domain under consideration; the small parameter $\varepsilon$ is put to be equal $l / L$. In what follows, we use the notations $B: C=\operatorname{tr}\left(B C^{\top}\right)$, where $B$ and $C$ are tensors of the second rank; $\mathbf{D}(x, \mathbf{u})=\frac{1}{2}\left(\nabla \mathbf{u}+\nabla \mathbf{u}^{\top}\right)$ is the symmetric part of $\nabla \mathbf{u}$; and $\mathbf{I}$ is the unit tensor.

The movement of the mixture in $G$ is described for $t>0$ by the system

$$
\left(\frac{\chi_{0}^{\varepsilon}}{\bar{c}_{f}^{2}}+\frac{1-\chi_{0}^{\varepsilon}}{\left(\bar{c}_{s}^{(0)}\right)^{2}}\right) p+\nabla \cdot \mathbf{w}=0
$$

\footnotetext{
${ }^{*}$ E-mail: artur-gerus@mail.ru

${ }^{* *}$ E-mail: sv.a.gritsenko@gmail.com

****-mail: anvarbek@list.ru55
} 


$$
\begin{gathered}
\left(\rho_{f} \chi_{0}^{\varepsilon}+\left(1-\chi_{0}^{\varepsilon}\right) \rho_{s}^{(0)}\right) \frac{\partial^{2} \mathbf{w}}{\partial t^{2}}=\nabla \cdot \mathbf{P}^{(0)}+\rho^{\varepsilon} \mathbf{F}, \\
\mathbf{P}^{(0)}=\chi_{0}^{\varepsilon} \bar{\alpha}_{\mu} \mathbf{D}\left(x, \frac{\partial \mathbf{w}}{\partial t}\right)+\left(1-\chi_{0}^{\varepsilon}\right) \bar{\alpha}_{\lambda}^{(0)} \mathbf{D}(x, \mathbf{w})-p \mathbf{I},
\end{gathered}
$$

where $\chi_{0}^{\varepsilon}$ is the characteristic function of the liquid part $G_{f}^{\varepsilon}$ in $G: \chi_{0}^{\varepsilon}(\mathbf{x})=\chi_{0}(\mathbf{x} / \varepsilon)$. The elastic properties of the solid skeleton in $G_{s}^{\varepsilon}$ and $\Omega_{s}^{\varepsilon}$ are different; while the liquid is the same in $G_{f}^{\varepsilon}$ and $\Omega_{f}^{\varepsilon}$.

On the common boundary $S^{(0)}$, the continuity conditions are fulfilled for the displacement

$$
\lim _{x \rightarrow \mathbf{x}^{0}, \mathbf{x} \in G} \mathbf{w}(\mathbf{x}, t)=\lim _{\mathbf{x} \rightarrow \mathbf{x}^{0}, \mathbf{x} \in \Omega} \mathbf{w}(\mathbf{x}, t)
$$

and the normal component of the momenta

$$
\lim _{\mathbf{x} \rightarrow \mathbf{x}^{0}, \mathbf{x} \in G} \mathbf{P}^{(0)}(\mathbf{x}, t) \cdot \mathbf{n}\left(\mathbf{x}^{0}\right)=\lim _{\mathbf{x} \rightarrow \mathbf{x}^{0}, \mathbf{x} \in \Omega} \mathbf{P}(\mathbf{x}, t) \cdot \mathbf{n}\left(\mathbf{x}^{0}\right) .
$$

The formulation of the problem is completed with the homogeneous boundary conditions

$$
\mathbf{w}(\mathbf{x}, t)=0, \quad(\mathbf{x}, t) \in S_{T}=S \times(0, T),
$$

on the boundary $S=\partial Q$ and the homogeneous initial conditions

$$
\mathbf{w}(\mathbf{x}, 0)=\frac{\partial \mathbf{w}}{\partial t}(\mathbf{x}, 0)=0, \quad \mathbf{x} \in Q .
$$

Differential equations (1)-(10) adequately describe the physical processes in the domain consisting of two different continuous media; however, they cannot be used for computation because of the presence of rapidly oscillating coefficients there. Our goal is to derive some averaged equations that do not contain such coefficients. In order to take advantage of the available inequalities for periodic structures and other results of the homogenization theory [1-3] as well as the method of two-scale convergence [4], we introduce some simplifying geometric assumptions.

Proposition 1. (1) Let $\chi(\mathbf{y})$ be a 1-periodic function; let $Y_{s}=\{\mathbf{y} \in Y: \chi(\mathbf{y})=0\}$ be the solid part of the unit cube $Y=(0,1)^{3} \subset \mathbb{R}^{3}$; and let the liquid part $Y_{f}=\{\mathbf{y} \in Y: \chi(\mathbf{y})=1\}$ be the open complement of the solid part. Suppose that $\gamma=\partial Y_{f} \cap \partial Y_{s}$, and $\gamma$ is a Lipschitz continuous surface.

(2) The domain $E_{f}^{\varepsilon}$ is the periodic replication in $\mathbb{R}^{3}$ of $Y_{f}^{\varepsilon}=\varepsilon Y_{f}$, and the domain $E_{s}^{\varepsilon}$ is the periodic replication in $\mathbb{R}^{3}$ of $Y_{s}^{\varepsilon}=\varepsilon Y_{s}$.

(3) The porous space $\Omega_{f}^{\varepsilon} \subset \Omega=\Omega \cap E_{f}^{\varepsilon}$ is the periodic replication in $\Omega$ of $\varepsilon Y_{f}$; and the solid skeleton $\Omega_{s}^{\varepsilon} \subset \Omega=\Omega \cap E_{s}^{\varepsilon}$ is the periodic replication in $\Omega$ of $\varepsilon Y_{s}$. The Lipschitz boundary

$$
\Gamma^{\varepsilon}=\partial \Omega_{s}^{\varepsilon} \cap \partial \Omega_{f}^{\varepsilon}
$$

is periodic replication in $\Omega$ of $\varepsilon \gamma$.

(4) $Y_{s}$ and $Y_{f}$ are connected sets.

Proposition 2. The solid skeleton $\Omega_{s}^{\varepsilon}$ is a connected domain.

Proposition 3. The porous space $\Omega_{f}^{\varepsilon}$ is a connected domain.

Analogous assumptions are also made about the domain $G$.

Moreover, it is assumed that all dimensionless parameters depend on the small parameter $\varepsilon$ and the following (finite or infinite) limits exist

$$
\begin{array}{cc}
\lim _{\varepsilon \rightarrow 0} \bar{\alpha}_{\mu}(\varepsilon)=\mu_{0}, & \lim _{\varepsilon \rightarrow 0} \bar{\alpha}_{\lambda}(\varepsilon)=\lambda_{0}, \\
\lim _{\varepsilon \rightarrow 0} \frac{\bar{\alpha}_{\mu}}{\varepsilon^{2}}=\mu_{1}, & \lim _{\varepsilon \rightarrow 0} \frac{\bar{\alpha}_{\lambda}^{(0)}}{\varepsilon_{\lambda}^{2}}=\lambda_{1}, \quad \lim _{\varepsilon \rightarrow 0} \frac{\bar{\alpha}_{\lambda}^{(0)}}{\varepsilon^{2}}=\lambda_{1}^{(0)} .
\end{array}
$$

In our model, the liquid is weakly compressible; i.e., $\mu_{0}=0$. 
As always, we introduce the notion of generalized solution and prove the existence and uniqueness of such a solution. We use the notations of [5] for the function spaces.

Let $\zeta(\mathbf{x})$ be the characteristic function of $\Omega$ in $Q$ and

$$
\begin{gathered}
\rho_{(0)}^{\varepsilon}=(1-\zeta)\left(\rho_{f} \chi_{0}^{\varepsilon}+\left(1-\chi_{0}^{\varepsilon}\right) \rho_{s}^{(0)}\right)+\zeta\left(\rho_{f} \chi^{\varepsilon}+\left(1-\chi^{\varepsilon}\right) \rho_{s}\right), \\
\int_{Q_{T}}\left(|\mathbf{F}(\mathbf{x}, t)|^{2}+\left|\frac{\partial \mathbf{F}}{\partial t}(\mathbf{x}, t)\right|^{2}\right) d x d t=F^{2}<\infty .
\end{gathered}
$$

Definition. A pair of functions $\left\{\mathbf{w}^{\varepsilon}, p^{\varepsilon}\right\}$ such that

$$
\mathbf{w}^{\varepsilon} \in \stackrel{\circ}{\mathbf{W}}_{2}^{1,1}\left(Q_{T}\right), \quad p^{\varepsilon} \in L_{2}\left(Q_{T}\right),
$$

is called a generalized solution of (1)-(10) if they satisfy the continuity equation

$$
\left((1-\zeta)\left(\frac{\chi_{0}^{\varepsilon}}{\bar{c}_{f}^{2}}+\frac{1-\chi_{0}^{\varepsilon}}{\left(\bar{c}_{s}^{(0)}\right)^{2}}\right)+\zeta\left(\frac{\chi^{\varepsilon}}{\bar{c}_{f}^{2}}+\frac{1-\chi^{\varepsilon}}{\bar{c}_{s}^{2}}\right)\right) p^{\varepsilon}+\nabla \cdot \mathbf{w}^{\varepsilon}=0
$$

almost everywhere in $Q_{T}$ and

$$
\int_{Q_{T}} \rho_{(0)}^{\varepsilon}\left(\frac{\partial \mathbf{w}^{\varepsilon}}{\partial t} \frac{\partial \boldsymbol{\varphi}}{\partial t}+\mathbf{F} \cdot \boldsymbol{\varphi}\right) d x d t=\int_{Q_{T}}\left(\zeta \mathbf{P}+(1-\zeta) \mathbf{P}^{(0)}\right): \mathbf{D}(x, \boldsymbol{\varphi}) d x d t
$$

for all functions $\boldsymbol{\varphi}$ such that $\boldsymbol{\varphi} \in \stackrel{\circ}{\mathbf{W}}_{2}^{1,0}\left(Q_{T}\right), \frac{\partial \boldsymbol{\varphi}}{\partial t} \in \mathbf{L}_{2}\left(\Omega_{T}\right)$ and $\boldsymbol{\varphi}(\mathbf{x}, T)=0$ for $\mathbf{x} \in Q$.

\section{THEOREM OF EXISTENCE AND UNIQUENESS OF A GENERALIZED SOLUTION}

Theorem 1. For all $\varepsilon>0$, the problem (1)-(10) has the unique generalized solution $\left\{\mathbf{w}^{\varepsilon}, p^{\varepsilon}\right\}$ on an arbitrary time interval $[0, T]$ and

$$
\begin{aligned}
\max _{0<t<T} \int_{\Omega}\left(\left|p^{\varepsilon}(\mathbf{x}, t)\right|^{2}+\left|\frac{\partial \mathbf{w}^{\varepsilon}}{\partial t}(\mathbf{x}, t)\right|^{2}+\left(1-\chi^{\varepsilon}\right) \bar{\alpha}_{\lambda}\left|\mathbf{D}\left(x, \mathbf{w}^{\varepsilon}\right)\right|^{2}\right) d x \\
+\max _{0<t<T} \int_{G}\left(\left|p^{\varepsilon}(\mathbf{x}, t)\right|^{2}+\left|\frac{\partial \mathbf{w}^{\varepsilon}}{\partial t}(\mathbf{x}, t)\right|^{2}+\left(1-\chi_{0}^{\varepsilon}\right) \bar{\alpha}_{\lambda}^{(0)}\left|\mathbf{D}\left(x, \mathbf{w}^{\varepsilon}\right)\right|^{2}\right) d x \\
+\max _{0<t<T} \int_{\Omega}\left(\left|\frac{\partial p^{\varepsilon}}{\partial t}(\mathbf{x}, t)\right|^{2}+\left|\frac{\partial^{2} \mathbf{w}^{\varepsilon}}{\partial t^{2}}(\mathbf{x}, t)\right|^{2}+\left(1-\chi^{\varepsilon}\right) \bar{\alpha}_{\lambda}\left|\mathbf{D}\left(x, \frac{\partial \mathbf{w}^{\varepsilon}}{\partial t}\right)\right|^{2}\right) d x \\
+\max _{0<t<T} \int_{G}\left(\left|\frac{\partial p^{\varepsilon}}{\partial t}(\mathbf{x}, t)\right|^{2}+\left|\frac{\partial^{2} \mathbf{w}^{\varepsilon}}{\partial t^{2}}(\mathbf{x}, t)\right|^{2}+\left(1-\chi_{0}^{\varepsilon}\right) \bar{\alpha}_{\lambda}^{(0)}\left|\mathbf{D}\left(x, \frac{\partial \mathbf{w}^{\varepsilon}}{\partial t}\right)\right|^{2}\right) d x \\
+\int_{\Omega_{T}} \chi^{\varepsilon} \bar{\alpha}_{\mu}\left(\left|\mathbf{D}\left(x, \frac{\partial \mathbf{w}^{\varepsilon}}{\partial t}\right)\right|^{2}+\left|\mathbf{D}\left(x, \frac{\partial^{2} \mathbf{w}^{\varepsilon}}{\partial t^{2}}\right)\right|^{2}\right) d x d t \\
+\int_{0}^{T} \int_{G} \chi_{0}^{\varepsilon} \bar{\alpha}_{\mu}^{(0)}\left(\left|\mathbf{D}\left(x, \frac{\partial \mathbf{w}^{\varepsilon}}{\partial t}\right)\right|^{2}+\left|\mathbf{D}\left(x, \frac{\partial^{2} \mathbf{w}^{\varepsilon}}{\partial t^{2}}\right)\right|^{2}\right) d x d t \leq C_{0} F^{2},
\end{aligned}
$$

where the constant $C_{0}$ is independent of $\varepsilon, \bar{\alpha}_{\lambda}, \bar{\alpha}_{\lambda}^{(0)}$, and $\bar{\alpha}_{\mu}$. 
Proof. The a priori estimate (13) and the existence of the unique generalized solution are proved on the basis of the energy identities

$$
\begin{aligned}
& \frac{1}{2} \frac{d}{d t} \int_{\Omega}\left(\rho^{\varepsilon}\left|\frac{\partial \mathbf{w}^{\varepsilon}}{\partial t}\right|^{2}+\left(1-\chi^{\varepsilon}\right) \bar{\alpha}_{\lambda} \mathbf{D}\left(x, \mathbf{w}^{\varepsilon}\right): \mathbf{D}\left(x, \mathbf{w}^{\varepsilon}\right)+\left|p^{\varepsilon}\right|^{2}\right) d x \\
& +\frac{1}{2} \frac{d}{d t} \int_{G}\left(\rho_{s}^{(0)}\left|\frac{\partial \mathbf{w}^{\varepsilon}}{\partial t}\right|^{2}+\left(1-\chi_{0}^{\varepsilon}\right) \bar{\alpha}_{\lambda}^{(0)} \mathbf{D}\left(x, \mathbf{w}^{\varepsilon}\right): \mathbf{D}\left(x, \mathbf{w}^{\varepsilon}\right)+\frac{1}{\left(\bar{c}_{s}^{(0)}\right)^{2}}\left|p^{\varepsilon}\right|^{2}\right) d x \\
& +\int_{\Omega} \chi^{\varepsilon}\left(\bar{\alpha}_{\mu} \mathbf{D}\left(x, \frac{\partial \mathbf{w}^{\varepsilon}}{\partial t}\right): \mathbf{D}\left(x, \frac{\partial \mathbf{w}^{\varepsilon}}{\partial t}\right)\right) d x \\
& +\int_{G} \chi_{0}^{\varepsilon}\left(\bar{\alpha}_{\mu} \mathbf{D}\left(x, \frac{\partial \mathbf{w}^{\varepsilon}}{\partial t}\right): \mathbf{D}\left(x, \frac{\partial \mathbf{w}^{\varepsilon}}{\partial t}\right)\right) d x=\int_{Q} \rho_{(0)}^{\varepsilon} \mathbf{F} \cdot \frac{\partial \mathbf{w}^{\varepsilon}}{\partial t} d x ; \\
& \frac{1}{2} \frac{d}{d t} \int_{\Omega}\left(\rho^{\varepsilon}\left|\frac{\partial^{2} \mathbf{w}^{\varepsilon}}{\partial t^{2}}\right|^{2}+\left(1-\chi^{\varepsilon}\right) \bar{\alpha}_{\lambda} \mathbf{D}\left(x, \frac{\partial \mathbf{w}^{\varepsilon}}{\partial t}\right): \mathbf{D}\left(x, \frac{\partial \mathbf{w}^{\varepsilon}}{\partial t}\right)+\left|\frac{\partial p^{\varepsilon}}{\partial t}\right|^{2}\right) d x \\
& +\frac{1}{2} \frac{d}{d t} \int_{G}\left(\rho_{s}^{(0)}\left|\frac{\partial^{2} \mathbf{w}^{\varepsilon}}{\partial t^{2}}\right|^{2}+\left(1-\chi_{0}^{\varepsilon}\right) \bar{\alpha}_{\lambda}^{(0)} \mathbf{D}\left(x, \frac{\partial \mathbf{w}^{\varepsilon}}{\partial t}\right): \mathbf{D}\left(x, \frac{\partial \mathbf{w}^{\varepsilon}}{\partial t}\right)+\frac{1}{\left(\bar{c}_{s}^{(0)}\right)^{2}}\left|\frac{\partial p^{\varepsilon}}{\partial t}\right|^{2}\right) d x \\
& +\int_{\Omega} \chi^{\varepsilon}\left(\bar{\alpha}_{\mu} \mathbf{D}\left(x, \frac{\partial^{2} \mathbf{w}^{\varepsilon}}{\partial t^{2}}\right): \mathbf{D}\left(x, \frac{\partial^{2} \mathbf{w}^{\varepsilon}}{\partial t^{2}}\right)\right) d x \\
& +\int_{G} \chi_{0}^{\varepsilon}\left(\bar{\alpha}_{\mu} \mathbf{D}\left(x, \frac{\partial^{2} \mathbf{w}^{\varepsilon}}{\partial t^{2}}\right): \mathbf{D}\left(x, \frac{\partial^{2} \mathbf{w}^{\varepsilon}}{\partial t^{2}}\right)\right) d x=\int_{Q} \rho_{(0)}^{\varepsilon} \frac{\partial \mathbf{F}}{\partial t} \cdot \frac{\partial^{2} \mathbf{w}^{\varepsilon}}{\partial t^{2}} d x,
\end{aligned}
$$

which are derived by the substitution into the integral identity (12) of an explicit expression for the tensors $\mathbf{P}$ and $\mathbf{P}^{(0)}$ from the equations of state (3) and (6), multiplication of (12) by $\frac{\partial \mathbf{w}^{\varepsilon}(\mathbf{x}, t)}{\partial t}$ and integration by parts over $Q$.

Theorem 1 is proved.

\section{AVERAGING THE MODEL}

In the derivation of averaged equations, we apply the Nguetseng's method of the two-scale convergence (see [4] and [6]). For a specific type of a continuous medium the limit regimes as $\varepsilon \rightarrow 0$ are obtained in [6-11]. The major problem in proving the averaged equations in this particular case is concerned with the conditions on the common boundary $S^{(0)}$ between two poroelastic domains $G$ and $\Omega$. In this article, an averaged model is derived for the domain consisting of two weakly-deformable soils permeated by a system of pores which are filled with a viscous weakly-compressible liquid.

Theorem 2. Let $\left\{\mathbf{w}^{\varepsilon}, p^{\varepsilon}\right\}$ be a generalized solution of $(1)-(10)$,

$$
\lambda_{0}^{(0)}=0, \quad \lambda_{1}^{(0)}<\infty, \quad 0<\lambda_{0}<\infty, \quad \mu_{0}=0, \quad \mu_{1}<\infty,
$$

and let $\mathbf{w}_{s}^{\varepsilon}=\mathbf{E}_{\Omega_{s}^{\varepsilon}}\left(\mathbf{w}^{\varepsilon}\right)$ be the extension operator from $\Omega_{s}^{\varepsilon}$ to $\Omega$. Then, in $G_{T}$, the limits $\mathbf{v}=\frac{\partial \mathbf{w}}{\partial t}$ (velocity of the liquid) and $p$ (pressure) of the sequences $\left\{\frac{\partial \mathbf{w}^{\varepsilon}}{\partial t}\right\}$ and $\left\{p^{\varepsilon}\right\}$ satisfy the system of equations of acoustics consisting of the momentum balance equation in the form

$$
\mathbf{v}(\mathbf{x}, t)=\int_{0}^{t} \mathbf{B}_{0}^{(a)}\left(\mu_{1}, \lambda_{1}^{(0)} ; t-\tau\right) \cdot \nabla p(\mathbf{x}, \tau) d \tau+\mathbf{f}(\mathbf{x}, t)
$$


and the continuity equation

$$
\left(\frac{m}{\bar{c}_{f}^{2}}+\frac{1-m}{\left(\bar{c}_{s}^{(0)}\right)^{2}}\right) \frac{\partial p}{\partial t}+\nabla \cdot \mathbf{v}=0 .
$$

In $\Omega_{T}$, the limit functions $m p_{f}$ (pressure of the liquid), $\mathbf{w}^{(f)}$ (displacement of the liquid), and $\mathbf{w}_{s}$ (displacement of the solid skeleton) of the sequences $\left\{\zeta \chi^{\varepsilon} p^{\varepsilon}\right\},\left\{\zeta \chi^{\varepsilon} \mathbf{w}^{\varepsilon}\right\}$, and $\left\{\zeta \mathbf{w}_{s}^{\varepsilon}\right\}$ satisfy the system of averaged equations consisting of the continuity equation

$$
\frac{m}{c_{f}^{2}} p_{f}+\nabla \cdot \mathbf{w}^{(f)}=\mathbf{C}_{0}^{s}: \mathbf{D}\left(x, \boldsymbol{w}_{s}\right)+\frac{c_{0}^{s}}{\lambda_{0}} p_{f},
$$

the momentum balance equation for the solid skeleton

$$
\rho_{f} \frac{\partial^{2} \mathbf{w}^{(f)}}{\partial t^{2}}+\rho_{s}^{(0)} \frac{\partial^{2} \mathbf{w}_{s}}{\partial t^{2}}=\nabla \cdot\left(\lambda_{0} \mathfrak{N}_{2}^{s}: \mathbf{D}\left(x, \mathbf{w}_{s}\right)-p_{f} \mathbf{C}_{1}^{s}\right)+\hat{\rho} \mathbf{F}
$$

and the momentum balance equation for the liquid part

$$
-\int_{0}^{t} \mathbf{B}^{(f)}\left(\mu_{1}, \infty ; t-\tau\right) \cdot\left(\nabla p_{f}+\rho_{f}\left(\frac{\partial^{2} \boldsymbol{w}_{s}}{\partial \tau^{2}}-\mathbf{F}\right)\right)(\mathbf{x}, \tau) d \tau=\frac{\partial \mathbf{w}^{(f)}}{\partial t}-m \frac{\partial \mathbf{w}_{s}}{\partial t} .
$$

The formulation of the problem is completed with the homogeneous boundary conditions

$$
\begin{aligned}
\mathbf{v}(\mathbf{x}, t) \cdot \mathbf{n}(\mathbf{x}) & =0, & & \mathbf{x} \in \partial G \backslash S^{(0)}, & & t>0, \\
\mathbf{w}^{(f)}(\mathbf{x}, t) \cdot \mathbf{n}(\mathbf{x}) & =0, & & \mathbf{x} \in \partial \Omega \backslash S^{(0)}, & & t>0, \\
\mathbf{w}_{s}(\mathbf{x}, t) & =0, & & \mathbf{x} \in \partial \Omega \backslash S^{(0)}, & & t>0,
\end{aligned}
$$

the homogeneous initial conditions

$$
\begin{gathered}
p(\mathbf{x}, 0)=0, \quad \mathbf{x} \in G \\
\boldsymbol{w}^{(f)}(\mathbf{x}, 0)=\mathbf{w}_{s}(\mathbf{x}, 0)=\frac{\partial \mathbf{w}_{s}}{\partial t}(\mathbf{x}, 0)=0, \quad \mathbf{x} \in \Omega
\end{gathered}
$$

and the continuity conditions on the common boundary $S_{T}^{(0)}$

$$
\begin{aligned}
& \lim _{\mathbf{x} \rightarrow \mathbf{x}^{0}, \mathbf{x} \in G} \mathbf{v}(\mathbf{x}, t) \cdot \mathbf{n}\left(\mathbf{x}^{0}\right)=\lim _{\mathbf{x} \rightarrow \mathbf{x}^{0}, \mathbf{x} \in \Omega}\left(\mathbf{v}^{(f)}(\mathbf{x}, t)+(1-m) \frac{\partial \mathbf{w}_{s}}{\partial t}(\mathbf{x}, t)\right) \cdot \mathbf{n}\left(\mathbf{x}^{0}\right), \\
& -\lim _{\mathbf{x} \rightarrow \mathbf{x}^{0}, \mathbf{x} \in G} p(\mathbf{x}, t) \mathbf{n}\left(\mathbf{x}^{0}\right)=\lim _{\mathbf{x} \rightarrow \mathbf{x}^{0}, \mathbf{x} \in \Omega}\left(\lambda_{0} \mathfrak{N}_{2}^{s}: \mathbf{D}\left(x, \mathbf{w}_{s}(\mathbf{x}, t)\right)-p_{f} \mathbf{C}_{1}^{s}\right) \cdot \mathbf{n}\left(\mathbf{x}^{0}\right) .
\end{aligned}
$$

In (14)-(25), $m=\int_{Y} \chi(y) d y=\langle\chi(y)\rangle_{Y} ; \mathbf{n}\left(\mathbf{x}^{0}\right)$ is the normal to $S^{(0)}$ at $\mathbf{x}^{0} \in S^{(0)} ; \mathbf{n}(\mathbf{x})$ is the normal to $\partial Q$ at $\mathbf{x} \in \partial Q$; and $\hat{\rho}=m \rho_{f}+(1-m) \rho_{s}^{(0)}$.

In the theorem, we use the notation $\mathbf{w}_{s}^{\varepsilon}=\mathbf{E}_{\Omega_{s}^{\varepsilon}}\left(\mathbf{w}^{\varepsilon}\right)$, where $\mathbf{E}_{\Omega_{s}^{\varepsilon}}: \mathbf{W}_{2}^{1}\left(\Omega_{s}^{\varepsilon}\right) \rightarrow \mathbf{W}_{2}^{1}(\Omega)$ is the extension operator from $\Omega_{s}^{\varepsilon}$ to $\Omega$ such that $\mathbf{w}_{s}^{\varepsilon}=\mathbf{w}^{\varepsilon}$ in $\Omega_{s}^{\varepsilon} \times(0, T)$ and

$$
\int_{\Omega}\left|\mathbf{w}_{s}^{\varepsilon}\right|^{2} d x \leq C_{0} \int_{\Omega_{s}^{\varepsilon}}\left|\mathbf{w}^{\varepsilon}\right|^{2} d x, \quad \int_{\Omega}\left|\mathbf{D}\left(x, \mathbf{w}_{s}^{\varepsilon}\right)\right|^{2} d x \leq C_{0} \int_{\Omega_{s}^{\varepsilon}}\left|\mathbf{D}\left(x, \mathbf{w}^{\varepsilon}\right)\right|^{2} d x .
$$

The correctness of this extension is substantiated in [12]. 
Proof. The a priori estimate (13) obtained in Theorem 1 allows us to take the weak and two-scale limits as $\varepsilon \rightarrow 0$ following the Nguetseng's theorem [4] and the results of [6-11].

The two-scale limit $P(\mathbf{x}, t, \mathbf{y})$ of $\left\{p^{\varepsilon}\right\}$ is given by the formula

$$
(1-\zeta) p+\zeta \chi(\mathbf{y}) p_{f}(\mathbf{x}, t)+\zeta(1-\chi(\mathbf{y})) P_{s}(\mathbf{x}, t, \mathbf{y})
$$

For $\mu_{1}<\infty$,

$$
\mathbf{W}(\mathbf{x}, t, \mathbf{y})=\chi(\mathbf{y}) \mathbf{W}(\mathbf{x}, t, \mathbf{y})+(1-\chi(\mathbf{y})) \mathbf{w}_{s}(\mathbf{x}, t) .
$$

The two-scale limit of $\left\{\mathbf{w}_{s}^{\varepsilon}\right\}$ equals $\mathbf{w}_{s}(\mathbf{x}, t)$, whereas the two-scale limit of $\left\{\mathbf{D}\left(x, \mathbf{w}_{s}^{\varepsilon}\right)\right\}$ is equal to $\mathbf{D}\left(x, \mathbf{w}_{s}(\mathbf{x}, t)\right)+\mathbf{D}(y, \mathbf{U}(\mathbf{x}, t, \mathbf{y}))$. Thus, we obtain the limit continuity equation

$$
\left(\frac{m}{\bar{c}_{f}^{2}}+\frac{(1-m)}{\bar{c}_{s}^{2}}\right) \frac{\partial p}{\partial t}+\nabla \cdot \mathbf{v}=0, \quad \mathbf{x} \in G, \quad t>0,
$$

and the boundary condition $\mathbf{v} \cdot \mathbf{n}=0$ for $\mathbf{x} \in \partial G \backslash S^{(0)}$ and $t>0$. For $\mu_{1}<\infty$ and $\lambda_{1}^{(0)}<\infty$, we have $\mathbf{v}=\frac{\partial \mathbf{w}}{\partial t}$.

Using the embedding $\nabla p \in \mathbf{L}_{2}\left(\Omega_{T}\right), \nabla\left(\frac{\partial p}{\partial t}\right) \in \mathbf{L}_{2}\left(\Omega_{T}\right)$, we derive the microscopic momentum balance equation

$$
\begin{array}{r}
\rho(\mathbf{y}) \frac{\partial^{2} \mathbf{W}}{\partial t^{2}}=\nabla_{y} \cdot\left(\mu_{1} \chi^{(0)}(\mathbf{y}) \mathbf{D}\left(y, \frac{\partial \mathbf{W}}{\partial t}\right)+\lambda_{1}^{(0)}(1-\chi(\mathbf{y})) \mathbf{D}(y, \mathbf{W})-\Pi \mathbf{I}\right)-\nabla p+\rho(\mathbf{y}) \mathbf{F} \\
\mathbf{y} \in Y, \quad t>0
\end{array}
$$

where $\rho(\mathbf{y})=\rho_{f} \chi^{(0)}(\mathbf{y})+\rho_{s}^{(0)}\left(1-\chi^{(0)}(\mathbf{y})\right)$, and the microscopic continuity equation

$$
\nabla_{y} \cdot \mathbf{W}=0, \quad \mathbf{y} \in Y .
$$

These equations are closed with the homogeneous initial conditions

$$
\mathbf{W}(\mathbf{x}, \mathbf{y}, 0)=\frac{\partial \mathbf{W}}{\partial t}(\mathbf{x}, \mathbf{y}, 0)=0, \quad \mathbf{y} \in Y .
$$

We look for a solution in the form of a sum

$$
\begin{aligned}
\mathbf{W}(\mathbf{x}, t, \mathbf{y}) & =\sum_{i=1}^{3} \int_{0}^{t} \mathbf{W}^{(i)}(\mathbf{y}, t-\tau) \frac{\partial p}{\partial x_{i}}(\mathbf{x}, \tau) d \tau+\sum_{i=1}^{3} \int_{0}^{t} \mathbf{W}_{F}^{(i)}(\mathbf{y}, t-\tau) F_{i}(\mathbf{x}, \tau) d \tau \\
\Pi(\mathbf{x}, t, \mathbf{y}) & =\sum_{i=1}^{3} \int_{0}^{t} \Pi^{(i)}(\mathbf{y}, t-\tau) \frac{\partial p}{\partial x_{i}}(\mathbf{x}, \tau) d \tau+\sum_{i=1}^{3} \int_{0}^{t} \Pi_{F}^{(i)}(\mathbf{y}, t-\tau) F_{i}(\mathbf{x}, \tau) d \tau
\end{aligned}
$$

where $\mathbf{F}(\mathbf{x}, t)=\left(F_{1}(\mathbf{x}, t), F_{2}(\mathbf{x}, t), F_{3}(\mathbf{x}, t)\right)$.

In turn, $\left\{\mathbf{W}^{(i)}, \Pi^{(i)}\right\}$ and $\left\{\mathbf{W}_{F}^{(i)}, \Pi_{F}^{(i)}\right\}, i=1,2,3$, are solutions of the periodic initial boundary value problems in $Y$ for $t>0$ :

$$
\begin{gathered}
\rho(\mathbf{y}) \frac{\partial^{2} \mathbf{W}^{(i)}}{\partial t^{2}}=\nabla_{y} \cdot\left(\mu_{1} \chi^{(0)}(\mathbf{y}) \nabla_{y}\left(\frac{\partial \mathbf{W}^{(i)}}{\partial t}\right)+\lambda_{1}^{(0)}\left(1-\chi^{(0)}(\mathbf{y})\right) \nabla_{y} \mathbf{W}^{(i)}-\Pi^{(i)} \mathbf{I}\right), \\
\nabla_{y} \cdot \mathbf{W}^{(i)}=0, \\
\mathbf{W}^{(i)}(\mathbf{y}, 0)=0, \quad \rho(\mathbf{y}) \frac{\partial \mathbf{W}^{(i)}}{\partial t}(\mathbf{y}, 0)=-\mathbf{e}_{i}, \quad \mathbf{y} \in Y,
\end{gathered}
$$


and

$$
\begin{gathered}
\rho(\mathbf{y}) \frac{\partial^{2} \mathbf{W}_{F}^{(i)}}{\partial t^{2}}=\nabla_{y} \cdot\left(\mu_{1} \chi^{(0)}(\mathbf{y}) \nabla_{y}\left(\frac{\partial \mathbf{W}_{F}^{(i)}}{\partial t}\right)+\lambda_{1}^{(0)}\left(1-\chi^{(0)}(\mathbf{y})\right) \nabla_{y} \mathbf{W}_{F}^{(i)}-\Pi_{F}^{(i)} \mathbf{I}\right), \\
\nabla_{y} \cdot \mathbf{W}_{F}^{(i)}=0, \\
\mathbf{W}_{F}^{(i)}(\mathbf{y}, 0)=0, \quad \frac{\partial \mathbf{W}_{F}^{(i)}}{\partial t}(\mathbf{y}, 0)=\mathbf{e}_{i}, \quad \mathbf{y} \in Y,
\end{gathered}
$$

respectively. In this case,

$$
\begin{gathered}
\frac{\partial \mathbf{W}}{\partial t}=\sum_{i=1}^{3} \int_{0}^{t} \frac{\partial \mathbf{W}^{(i)}}{\partial t}(\mathbf{y}, t-\tau) \frac{\partial p}{\partial x_{i}}(\mathbf{x}, \tau) d \tau+\sum_{i=1}^{3} \int_{0}^{t} \frac{\partial \mathbf{W}_{F}^{(i)}}{\partial t}(\mathbf{y}, t-\tau) F_{i}(\mathbf{x}, \tau) d \tau, \\
\frac{\partial \mathbf{w}}{\partial t}=\sum_{i=1}^{3} \int_{0}^{t}\left\langle\frac{\partial \mathbf{W}^{(i)}}{\partial t}\right\rangle_{Y}(t-\tau) \frac{\partial p}{\partial x_{i}}(\mathbf{x}, \tau) d \tau+\sum_{i=1}^{3} \int_{0}^{t}\left\langle\frac{\partial \mathbf{W}_{F}^{(i)}}{\partial t}\right\rangle_{Y}(\mathbf{y}, t-\tau) F_{i}(\mathbf{x}, \tau) d \tau \\
=\int_{0}^{t} \mathbf{B}_{0}^{(a)}\left(\mu_{1}, \lambda_{1}^{(0)} ; t-\tau\right) \cdot \nabla p(\mathbf{x}, \tau) d \tau+\mathbf{f}(\mathbf{x}, t),
\end{gathered}
$$

where

$$
\begin{gathered}
\mathbf{B}_{0}^{(a)}\left(\mu_{1}, \lambda_{2}^{(0)} ; t\right)=\sum_{i=1}^{3}\left\langle\frac{\partial \mathbf{W}^{(i)}}{\partial t}\right\rangle_{Y}(t) \otimes \mathbf{e}_{i}, \\
\mathbf{f}(\mathbf{x}, t)=\sum_{i=1}^{3} \int_{0}^{t}\left\langle\frac{\partial \mathbf{W}_{F}^{(i)}}{\partial t}\right\rangle_{Y}(\mathbf{y}, t-\tau) F_{i}(\mathbf{x}, \tau) d \tau .
\end{gathered}
$$

Here the matrix $\mathbf{a} \otimes \mathbf{b}$ is defined as $(\mathbf{a} \otimes \mathbf{b}) \cdot \mathbf{c}=\mathbf{a}(\mathbf{b} \cdot \mathbf{c})$ for arbitrary vectors $\mathbf{a}, \mathbf{b}$, and $\mathbf{c}$.

Put

$$
\mathfrak{N}^{(0)}=\sum_{i, j=1}^{3} \mathbf{J}^{i j} \otimes \mathbf{J}^{i j}+\frac{c_{s}^{2}}{\lambda_{0}} \mathbf{I} \otimes \mathbf{I}
$$

where $\mathbf{J}^{i j}=\frac{1}{2}\left(e_{i} \otimes e_{j}+e_{j} \otimes e_{i}\right)$; the tensor $A \otimes B$ of the fourth rank is defined as follows: $(A \otimes B)$ : $C=A(B: C)$ for every tensor $C$ of the second rank.

The functions $\mathbf{U}_{2}^{(i j)}(\mathbf{y})$ and $\mathbf{U}_{2}^{(0)}(\mathbf{y})$ are solutions of the periodic problems

$$
\begin{gathered}
\nabla_{y} \cdot\left((1-\chi)\left(\mathfrak{N}^{(0)}:\left(\mathbf{J}^{(i j)}+\mathbf{D}\left(y, \mathbf{U}_{2}^{(i j)}\right)\right)\right)\right)=0 \quad \text { in } Y, \\
\nabla_{y} \cdot\left((1-\chi)\left(\mathfrak{N}^{(0)}: \mathbf{D}\left(y, \mathbf{U}_{2}^{(0)}\right)+\mathbf{I}\right)\right)=0 \quad \text { in } Y .
\end{gathered}
$$

Put

$$
\mathbf{U}(\mathbf{x}, t, \mathbf{y})=\sum_{i, j=1}^{3} \mathbf{U}_{2}^{(i j)}(\mathbf{y}) D_{i j}(\mathbf{x}, t)
$$

Then

$$
\langle\mathbf{D}(y, \mathbf{U})\rangle_{Y_{s}}=\sum_{i, j=1}^{3}\left\langle\mathbf{D}\left(y, \mathbf{U}_{2}^{(i j)}\right)\right\rangle_{Y_{s}} D_{i j}=\left(\sum_{i, j=1}^{3}\left\langle\mathbf{D}\left(y, \mathbf{U}_{2}^{(i j)}\right)\right\rangle_{Y_{s}} \otimes \mathbf{J}^{(i j)}\right): \mathbf{D}\left(x, \mathbf{w}_{s}\right),
$$


and

$$
\begin{gathered}
\mathfrak{N}_{2}^{s}=\mathfrak{N}^{(0)}:\left((1-m) \sum_{i, j=1}^{3} \mathbf{J}^{i j} \otimes \mathbf{J}^{i j}+\sum_{i, j=1}^{3}\left\langle\mathbf{D}\left(y, \mathbf{U}_{2}^{(i j)}\right)\right\rangle_{Y_{s}} \otimes \mathbf{J}^{(i j)}\right), \\
\mathbf{C}_{1}^{s}=m \mathbf{I}-\left\langle\mathbf{D}\left(y, \mathbf{U}_{2}^{(0)}\right)\right\rangle_{Y_{s}} .
\end{gathered}
$$

Using (28), we obtain

$$
\left\langle\nabla_{y} \cdot \mathbf{U}\right\rangle_{Y_{s}}=\sum_{i, j=1}^{3}\left\langle\nabla_{y} \cdot \mathbf{U}_{2}^{(i j)}\right\rangle_{Y_{s}} D_{i j}=\left(\sum_{i, j=1}^{3}\left\langle\nabla_{y} \cdot \mathbf{U}_{2}^{(i j)}\right\rangle_{Y_{s}} \mathbf{J}^{i j}\right): \mathbf{D}\left(x, \mathbf{w}_{s}\right),
$$

therefore,

$$
\mathbf{C}_{0}^{s}=\sum_{i, j=1}^{3}\left\langle\nabla_{y} \cdot \mathbf{U}_{2}^{(i j)}\right\rangle_{Y_{s}} \mathbf{J}^{i j}, \quad c_{0}^{s}=\left\langle\nabla_{y} \cdot \mathbf{U}_{2}^{(0)}\right\rangle_{Y_{s}}
$$

Consider the case $\mu_{1}>0$ :

$$
\begin{gathered}
\mathbf{W}^{(f)}=\mathbf{w}_{s}(\mathbf{x}, t)+\sum_{i=1}^{3} \int_{0}^{t} \mathbf{W}_{i}^{(f)}(\mathbf{y}, t-\tau)\left(\frac{\partial p}{\partial x_{i}}(\mathbf{x}, \tau)+\rho_{f} \frac{\partial^{2} \mathbf{w}_{s}}{\partial \tau^{2}}(\mathbf{x}, \tau)\right) d \tau \\
\Pi^{(f)}(\mathbf{x}, t, \mathbf{y})=\sum_{i=1}^{3} \int_{0}^{t} \Pi_{i}^{(f)}(\mathbf{y}, t-\tau)\left(\frac{\partial p}{\partial x_{i}}(\mathbf{x}, \tau)+\rho_{f} \frac{\partial^{2} \mathbf{w}_{s}}{\partial \tau^{2}}(\mathbf{x}, \tau)\right) d \tau
\end{gathered}
$$

where $\left\{\mathbf{W}_{i}^{(f)}, \Pi_{i}^{(f)}\right.$ big $\}, i=1,2,3$, are solutions of the following periodic initial boundary value problems:

$$
\begin{gathered}
\rho_{f} \frac{\partial^{2} \mathbf{W}_{i}^{(f)}}{\partial t^{2}}=\frac{\mu_{1}}{2} \Delta_{y}\left(\frac{\partial \mathbf{W}_{i}^{(f)}}{\partial t}\right)-\nabla_{y} \Pi_{i}^{(f)}, \quad(\mathbf{y}, t) \in Y_{f} \times(0, T), \\
\nabla_{y} \cdot \mathbf{W}_{i}^{(f)}(\mathbf{y}, t)=0, \quad(\mathbf{y}, t) \in Y_{f} \times(0, T), \\
\mathbf{W}_{i}^{(f)}(\mathbf{y}, 0)=0, \quad \rho_{f} \frac{\partial \mathbf{W}_{i}^{(f)}}{\partial t}(\mathbf{y}, 0)=\mathbf{e}_{i}, \quad \mathbf{y} \in Y_{f}, \\
\mathbf{W}_{i}^{(f)}(\mathbf{y}, t)=0, \quad(\mathbf{y}, t) \in \gamma \times(0, T),
\end{gathered}
$$

for almost all $\mathrm{x} \in \Omega_{T}$.

By definition,

$$
\begin{aligned}
& \frac{\partial \mathbf{w}^{(f)}}{\partial t}(\mathbf{x}, t)=\int_{Y_{f}} \frac{\partial \mathbf{W}^{(f)}}{\partial t}(\mathbf{x}, t, \mathbf{y}) d y \\
& =m \frac{\partial \mathbf{w}_{s}}{\partial t}-\int_{0}^{t}\left(\sum_{i=1}^{3}\left(\int_{Y_{f}} \frac{\partial \mathbf{W}_{i}^{(f)}}{\partial t}(\mathbf{y}, t-\tau) d y\right) \otimes \mathbf{e}_{i}\right) \cdot\left(\nabla p(\mathbf{x}, \tau)+\rho_{f} \frac{\partial^{2} \mathbf{w}_{s}}{\partial \tau^{2}}(\mathbf{x}, \tau)\right) d \tau \\
& \quad=m \frac{\partial \mathbf{w}_{s}}{\partial t}-\int_{0}^{t} \mathbf{B}^{(f)}\left(\mu_{1}, \infty ; t-\tau\right) \cdot\left(\nabla p(\mathbf{x}, \tau)+\rho_{f} \frac{\partial^{2} \mathbf{w}_{s}}{\partial \tau^{2}}(\mathbf{x}, \tau)\right) d \tau,
\end{aligned}
$$


where

$$
\mathbf{B}^{(f)}\left(\mu_{1}, \infty ; t\right)=\sum_{i=1}^{3}\left(\int_{Y_{f}} \frac{\partial \mathbf{W}_{i}^{(f)}}{\partial t}(\mathbf{y}, t) d y\right) \otimes \mathbf{e}_{i} .
$$

Consider now the case $\mu_{1}=0$. For $\mu_{1}=0$, the microscopic momentum balance equation for the liquid component has the form

$$
\rho_{f} \frac{\partial^{2} \mathbf{W}^{(f)}}{\partial t^{2}}=-\nabla_{y} \Pi^{(f)}-\nabla p
$$

The boundary condition on $\gamma$ has the form

$$
\left(\mathbf{W}^{(f)}(\mathbf{x}, t, \mathbf{y})-\mathbf{w}_{s}(\mathbf{x}, t)\right) \cdot \mathbf{n}(\mathbf{y})=0
$$

and is a corollary of the microscopic continuity equation and the representation

$$
\mathbf{W}(\mathbf{x}, t, \mathbf{y})=\chi(\mathbf{y}) \mathbf{W}^{(f)}(\mathbf{x}, t, \mathbf{y})+(1-\chi(\mathbf{y})) \mathbf{w}_{s}(\mathbf{x}, t), \quad \mathbf{y} \in Y .
$$

To solve (32), we apply the operator $\left(\nabla_{y}, \cdot\right)$ :

$$
0=\nabla_{y} \cdot\left(\rho_{f} \frac{\partial^{2} \mathbf{W}^{(f)}}{\partial t^{2}}\right)=-\nabla_{y} \cdot\left(\nabla_{y} \Pi^{(f)}\right) .
$$

Then (33) and (32) yield the boundary condition for the pressure $\Pi^{(f)}$ on $\gamma$ :

$$
\nabla_{y} \Pi^{(f)} \cdot \mathbf{n}(\mathbf{y})=-\left(\nabla p+\rho_{f} \frac{\partial^{2} \boldsymbol{w}_{s}}{\partial t^{2}}\right) \cdot \mathbf{n}(\mathbf{y})
$$

Let

$$
\Pi^{(f)}=-\left(\sum_{i=1}^{3} \Pi_{i}^{(f)}(\mathbf{y}) \mathbf{e}_{i}\right) \cdot\left(\nabla p+\varrho_{f} \frac{\partial^{2} \mathbf{w}_{s}}{\partial t^{2}}\right),
$$

where $\Pi_{i}^{(f)}, i=1,2,3$, are solutions of the periodic initial boundary value problems

$$
\Delta_{y} \Pi_{i}^{(f)}=0, \quad \mathbf{y} \in Y_{f}, \quad\left(\nabla_{y} \Pi_{i}^{(f)}-\mathbf{e}_{i}\right) \cdot \mathbf{n}(\mathbf{y})=0, \quad \mathbf{y} \in \gamma .
$$

Then

$$
\nabla_{y} \Pi^{(f)}=-\left(\sum_{i=1}^{3} \nabla_{y} \Pi_{i}^{(f)} \otimes \mathbf{e}_{i}\right) \cdot\left(\nabla p+\rho_{f} \frac{\partial^{2} \mathbf{w}_{s}}{\partial t^{2}}\right) .
$$

After integrating (32) over $Y_{f}$, we arrive at the momentum balance equation for the liquid component

$$
\rho_{f} \mathbf{B}^{(f)}(0, \infty)=m \mathbf{I}-\left(\sum_{i=1}^{3} \int_{Y_{f}} \nabla_{y} \Pi_{i}^{(f)}(\mathbf{y}) d y \otimes \mathbf{e}_{i}\right) .
$$

The proof of Theorem 2 is complete.

\section{ACKNOWLEDGMENTS}

The authors were supported by the Russian Foundation for Basic Research (project no. 14-1700556). 


\section{REFERENCES}

1. E. Sanchez-Palencia, Non-Homogeneous Media and Vibration Theory (Springer, Berlin, 1980).

2. N. S. Bakhvalov and G. P. Panasenko, Homogenisation: Averaging Processes in Periodic Media. Mathematical Problems in the Mechanics of Composite Materials (Nauka, Moscow, 1984; Kluwer Academic Publ., Dordrecht, 1989).

3. V. V. Jikov, S. M. Kozlov, and O. A. Oleinik, Homogenization of Differential Operators and Integral Functionals (Nauka, Moscow, 1993; Springer, New York, 1994).

4. D. Lukkassen, G. Nguetseng, and P. Wall, "Two-Scale Convergence," Internat. J. Pure Appl. Math. 2 (1), 35-86 (2002).

5. O. A. Ladyženskaja, V. A. Solonnikov, and N. N. Ural'ceva, Linear and Quasi-Linear Equations of Parabolic Type (Nauka, Moscow, 1967; AMS, 1968).

6. A. Meirmanov, "Nguetseng's Two-Scale Convergence Method for Filtration and Seismic Acoustic Problems in Elastic Porous Media," Sibir. Mat. Zh. 48 (3), 64-67 (2007) [Siberian Math. J. 48 (3), 519-538 (2007)].

7. A. Meirmanov, "Homogenized Models for Filtration and for Acoustic Wave Propagation in Thermo-Elastic Porous Media," Europ. J. Appl. Math. 19, 259-284 (2008).

8. A. Meirmanov, "A Description of Seismic Acoustic Wave Propagation in Porous Media via Homogenization," SIAM J. Math. Anal. 40 (3), 1272-1289 (2008).

9. A. Meirmanov, "Derivation of the Equations of Nonisothermal Acoustics in Elastic Porous Media," Sibir. Mat. Zh. 51 (1), 156-174 (2010) [Siberian Math. J. 51 (1), 128-143 (2010)].

10. A. Meirmanov, "Acoustics Equations in Poroelastic Media," Sibir. Zh. Industr. Mat. 13 (2), 98-110 (2010) [J. Appl. Indust. Math. 5(2), 247-258 (2011)].

11. A. Meirmanov, "Derivation of Equations of Seismic and Acoustic Wave Propagation and Equations of Filtration via Homogenization of Periodic Structures,” J. Math. Sci. 163 (2), 111-172 (2009).

12. C. Conca, "On the Application of the Homogenization Theory to a Class of Problems Arising in Fluid Mechanics," Math. Pures Appl. 64, 31-75 (1985). 